\title{
An Autopsy Case of Pulmonary Veno-Occlusive Disease Complicated with Chronic Obstructive Pulmonary Disease and Severe Pulmonary Hypertension
}

\author{
Takaaki Suzuki, ${ }^{1,2}$ MD, Kimihiko Hirose, ${ }^{1}$ MD, Fumiko Tabei, ${ }^{1}$ MD, Yasuyuki Sugishita, ${ }^{1}$ MD, \\ Teruaki Oka, ${ }^{3}$ MD, Satoshi Ishii, ${ }^{2}$ MD, Takayuki Fujiwara, ${ }^{2}$ MD, Norifumi Takeda, ${ }^{2}$ MD, \\ Issei Komuro, ${ }^{2} \mathrm{MD}$ and Nobuhiko Itoh, ${ }^{1} \mathrm{MD}$
}

\begin{abstract}
Summary
Chronic obstructive pulmonary disease (COPD) is a chronic inflammatory lung disease with obstructed airflow and frequently causes secondary mild-moderate pulmonary hypertension $(\mathrm{PH})$. However, a low proportion $(1 \%-5 \%)$ of COPD patients develop severe therapy-resistant $\mathrm{PH}$, and it is crucial to determine whether the patient has another disease capable of causing severe $\mathrm{PH}$, including pulmonary arterial hypertension.

Here, we describe a case of a 71-year-old male with COPD complicated by severe PH and right heart failure. He had a history of heavy smoking and developed progressive hypoxemia on exertion. He had severe airflow limitation (forced expiratory volume $\%$ in one second, FEV $1.0 \%=42.8 \%$ ) with a markedly reduced diffusing capacity of the lung (predicted diffusion capacity of carbon monoxide, \%DLCO $=29 \%$ ), and highresolution computed tomography (CT) demonstrated significant lung parenchymal abnormalities such as diffuse interlobular septal thickening, ground-glass opacities, and enlarged mediastinal lymph nodes. He was diagnosed with group $3 \mathrm{PH}$ caused by COPD but resistant to the treatment of COPD, diuretics, and oxygen therapy. Pathohistological analysis of autopsy specimens revealed the coexistence of interstitial fibrosis and partial occlusion of the small intrapulmonary veins, which led to a conclusive diagnosis of pulmonary veno-occlusive disease (PVOD).

Because of its rarity and similarity with idiopathic pulmonary arterial hypertension, PVOD is difficult to diagnose antemortem and has a poor prognosis. High-resolution CT findings (septal thickening, ground glass, and enlarged lymph nodes) and severely reduced DLCO should be carefully evaluated for the early detection and treatment of PVOD in COPD patients with severe PH.
\end{abstract}

Key words: Postmortem autopsy, High-resolution computed tomography, DLCO

(Int Heart J 2021; 62: 1186-1190)

$\mathrm{P}$ ulmonary hypertension $(\mathrm{PH})$ is a refractory cardiopulmonary disease characterized by increased pulmonary arterial pressure and pulmonary vascular resistance, which lead to fatal respiratory and circulatory failure. $\mathrm{PH}$ is caused by various underlying diseases and divided into the following categories by the World Health Organization $(\mathrm{WHO})^{1)}$ : group 1, pulmonary arterial hypertension $(\mathrm{PAH})$; group 2, $\mathrm{PH}$ due to left heart disease; group 3, PH due to lung disease; group 4, $\mathrm{PH}$ due to chronic blood clots in the lungs; group 5, PH due to unknown causes. Chronic obstructive pulmonary disease (COPD) is a chronic inflammatory lung disease that causes obstructed airflow from the lungs, ${ }^{2)}$ and group 3 $\mathrm{PH}$ is a frequent and important complication of COPD $(10 \%-90 \%))^{3)}$ The mechanisms of development of $\mathrm{PH}$ in COPD (COPD-PH) include hypoxic pulmonary arterial vasoconstriction, pulmonary vascular bed destruction, compression of alveolar vessels by emphysematous lung hyperinflation, endothelial dysfunction and remodeling provoked by irritating gas and particulate matter, most often from cigarette smoke. ${ }^{4)}$

Generally, COPD-PH shows mild to moderately elevated pulmonary arterial pressure. ${ }^{5)}$ Nevertheless, a small group of patients with moderate COPD develop severe $\mathrm{PH}$ classified as "out-of-proportion PH," and it is uncertain whether out-of-proportion PH is caused merely by COPD or by a combination of the other PH-causing etiologies, including group $1 \mathrm{PH}$ diseases.) Here, we describe a case of a 71-year-old male with out-of-proportion $\mathrm{PH}$, in which postmortem autopsy revealed the coexistence of COPD and pulmonary veno-occlusive disease (PVOD), a group 1 PH. PVOD has characteristics of hypoxemia on exertion, reduced DLCO and high-resolution computed tomography (CT) findings such as septal thickening, ground glass, and enlarged lymph nodes. Although the coexistence of COPD and PVOD is rare and difficult to find, cautious evaluation

From the 'Department of Cardiovascular Medicine, Kanto Central Hospital of the Mutual Aid Association of Public School Teachers, Tokyo, Japan, ${ }^{2}$ Department of Cardiovascular Medicine, The University of Tokyo Hospital, Tokyo, Japan and ${ }^{3}$ Department of Pathology, Fukujuuji Hospital, Tokyo, Japan.

Address for correspondence: Takaaki Suzuki, MD, Department of Cardiovascular Medicine, The University of Tokyo Hospital, 7-3-1 Hongo, Bunkyo-ku, Tokyo 113-8655, Japan. E-mail: tottipilrocosta@yahoo.co.jp

Received for publication March 5, 2021. Revised and accepted May 7, 2021.

doi: 10.1536/ihj.21-133

All rights reserved by the International Heart Journal Association. 

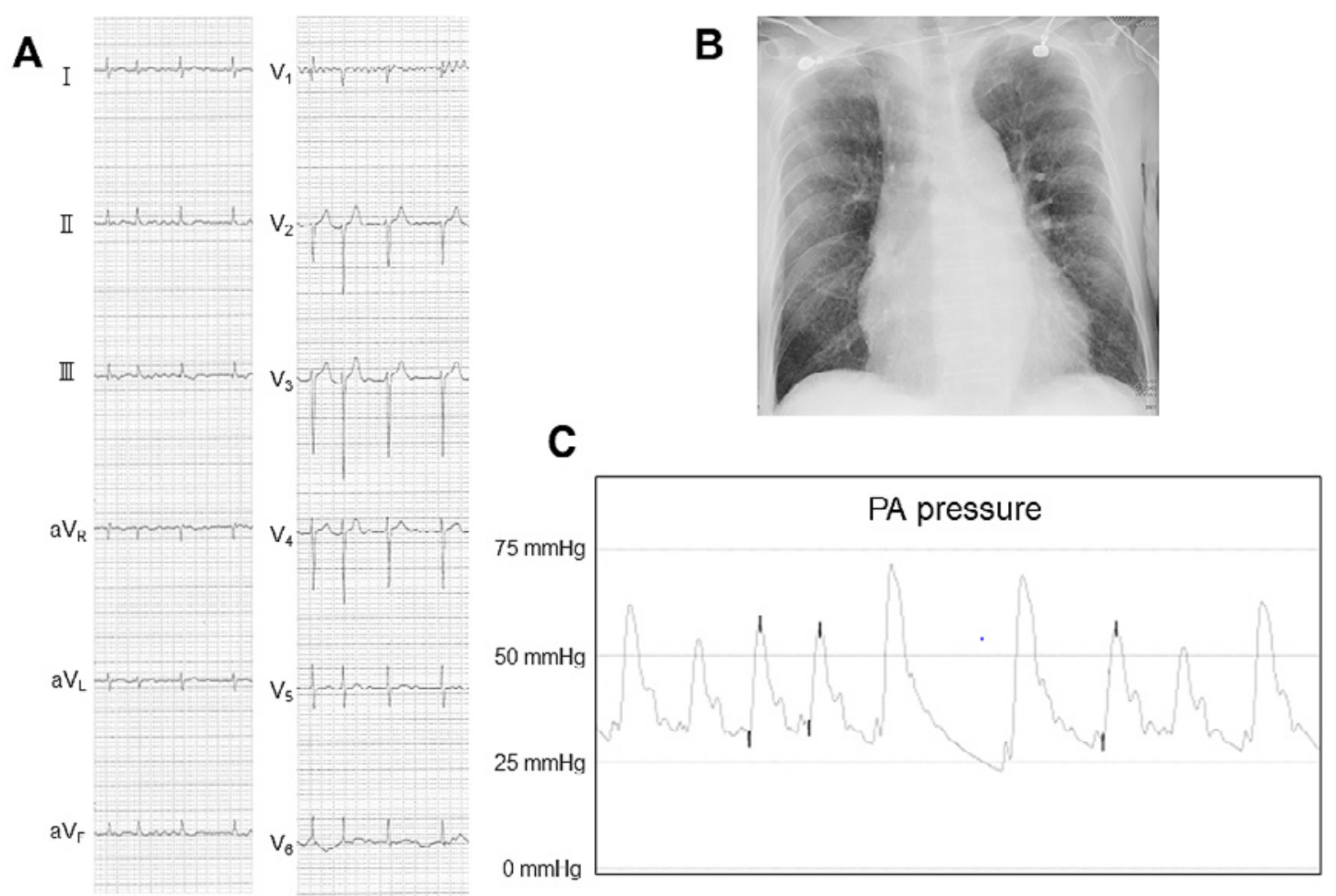

Figure 1. Electrocardiography, Chest X-ray, and pulmonary arterial pressure at the first admission. A: Electrocardiography showing atrial fibrillation, clockwise rotation, and low voltage in limb leads. B: Chest X-ray. C: Systolic pulmonary arterial pressure (PAP), diastolic PAP, and mean PAP were $56 \mathrm{mmHg}, 30 \mathrm{mmHg}$, and $39 \mathrm{mmHg}$, respectively.

of these findings is the key to detecting PVOD in COPD patients.

\section{Case Report}

The patient was a 71-year-old male ex-smoker with a Brinkman index of 3,000 who had a medical history of lung emphysema, atrial fibrillation, diabetes mellitus, and hyperuricemia. He developed progressive dyspnea (New York Heart Association [NYHA] functional capacity class III) and leg edema and presented to a local hospital. He was diagnosed as having pneumonia and right heart failure, and treatment with antibiotics and diuretics was initiated.

He showed little improvement in symptoms despite 2 months of the treatment and was thus, transferred to our hospital for further evaluation and treatment with NYHA class IV. Peripheral oxygen saturation $\left(\mathrm{SpO}_{2}\right)$ was $93 \%$ with nasal oxygen at $2 \mathrm{~L} /$ minute. Electrocardiography revealed atrial fibrillation, clockwise rotation, and low voltage in limb leads (Figure 1A). Chest roentgenogram identified cardiomegaly (Figure 1B), and his plasma brain-type natriuretic peptide (BNP) concentration was elevated (100 $\mathrm{pg} / \mathrm{mL}$ ).

Transthoracic echocardiography showed a normal left ventricular systolic function with an ejection fraction of $72 \%$, a dilated right ventricle, moderate tricuspid regurgitation with a peak pressure gradient of $44 \mathrm{mmHg}$, and a collapsed inferior vena cava. A respiratory function test revealed significantly low forced expiratory volume $1 \mathrm{sec}-$ ond/forced vital capacity (FEV1.0\%), diffusing capacity of the lung for carbon monoxide (\%DLCO), and the diffusing capacity divided by alveolar volume (\%DLCO/VA), which was $42.8 \%, 29 \%$, and $27 \%$, respectively. On cardiac catheterization, mean pulmonary artery pressure (mPAP) and pulmonary vascular resistance markedly increased to $39 \mathrm{mmHg}$ (Figure 1C) and 13.5 Wood units, respectively. The cardiac index calculated by the Fick method decreased to $1.5 \mathrm{~L} /$ minute $/ \mathrm{m}^{2}$ with a normal mean pulmonary artery wedge pressure (mPAWP) of $6 \mathrm{mmHg}$, and coronary angiography detected no visible stenosis. A chest CT scan showed diffuse emphysema, interlobular septal thickening, ground-glass opacities, and enlarged mediastinal lymph nodes in the lung (Figure 2). There were no CT findings indicating significant lung fibrosis such as reticulation, honeycombing, and traction bronchiectasis. Pulmonary ventilation/perfusion scintigraphy showed normal findings and did not reveal perfusion defect or ventilation/perfusion mismatch indicating pulmonary embolism.

The treatment with diuretics, oxygen therapy, and inhaling bronchodilator was continued because of the clinical diagnosis of severe PH caused by COPD. However, his respiratory status further deteriorated; $\mathrm{SpO}^{2}$ during the 6-minute walk test with nasal oxygen at $2 \mathrm{~L} /$ minute decreased from $95 \%$ to $87 \%$, and the 6-minute walk distance was only $103 \mathrm{~m}$. Therefore, we suspected that his severe COPD-PH might be caused by other PH etiologies. After excluding the possibility of group 2 and $4 \mathrm{PH}$ according to the prior examinations described above, bera- 

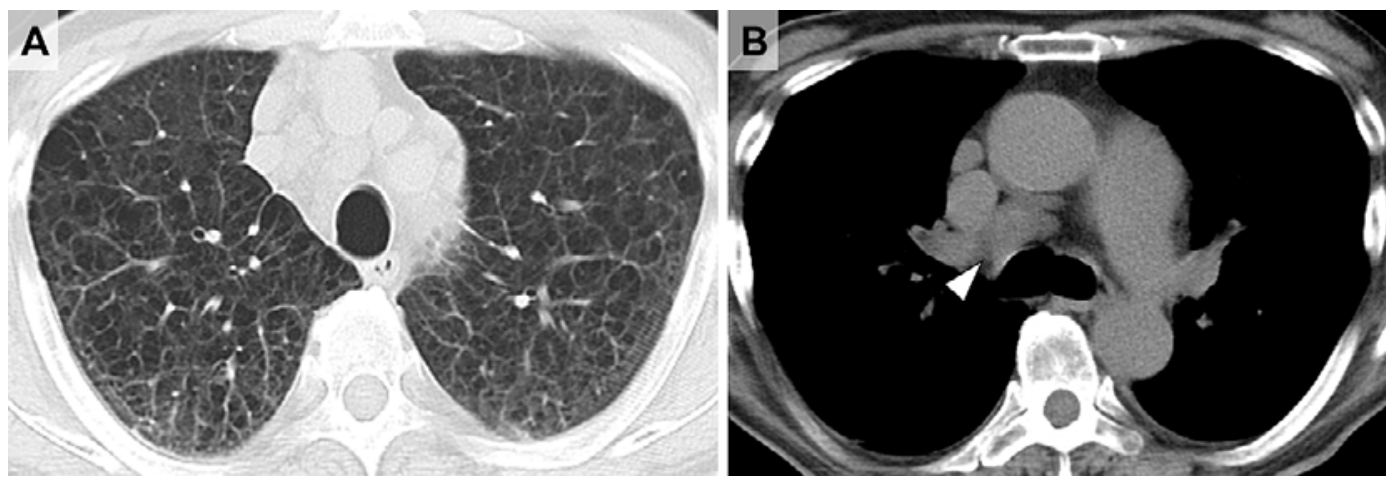

Figure 2. Chest computed tomography (CT). A: High-resolution CT showed diffuse lung emphysema, patchy ground-glass opacities, and a thickened lung lobe septum. B: The arrowhead indicates enlarged mediastinal lymph nodes.

prost sodium, a prostacyclin derivative, was started at a dose of $120 \mu \mathrm{g} / \mathrm{day}$ as a group $1 \mathrm{PH}$-targeted therapy on the twentieth hospital day. However, we discontinued it two days after the first administration because of the facial flushing and abdominal distension. The patient started on home oxygen therapy and was discharged on the thirtieth hospital day, and $\mathrm{SpO}_{2}$ was $97 \%$ with nasal oxygen at $2 \mathrm{~L} /$ minute.

About two weeks later, he was re-hospitalized because of worsening of dyspnea on exertion and deteriorations of $\mathrm{SpO}_{2}$ and arterial oxygen pressure of $84 \%$ and 56 $\mathrm{mmHg}$, respectively, at $2 \mathrm{~L} /$ minute oxygen via a nasal cannula. He gained weight with marked leg edema, and plasma BNP concentration further increased to $405 \mathrm{pg} /$ $\mathrm{mL}$. Echocardiography showed a newly emerged Dshaped left ventricle and moderate tricuspid valve regurgitation with a peak pressure gradient of $74 \mathrm{mmHg}$, indicating the progression of $\mathrm{PH}$ and right heart failure. Despite aggressive diuretics and noninvasive positive pressure ventilation therapy, his clinical status remained NYHA class III/IV. He developed pneumonia on the twentieth hospital day and died on the thirty-third day.

We performed a postmortem autopsy to examine the cause of severe respiratory failure. Gross pathologic findings were marked dilatation of the right atrium and ventricle, wall thickening and proliferative trabeculae carneae of the right ventricle, and lung emphysema. A pathohistological analysis of the lungs showed advanced destructive images of alveoli and interstitial fibrosis. There were no thrombi in pulmonary arterioles or abnormal pulmonary arterial wall thickening. However, we found fibrotic stenosis, intimal thickening, and partial occlusion of the small intrapulmonary veins and pre-septal venules (Figure 3 ), and the final autopsy diagnosis was PVOD with COPD.

\section{Discussion}

$\mathrm{PH}$ is a frequent and critical complication of COPD. Studies of spirometric Global Initiative for Chronic Obstructive Lung Disease (GOLD) stage IV patients showed that up to $90 \%$ of patients present with high mPAP (> 20 $\mathrm{mmHg}$ ) and COPD-PH is associated with worse mortality, symptoms such as dyspnea, and needs of greater health resources. ${ }^{7)}$ Generally, most of COPD-PH shows mild to moderate elevation in MPAP ranging between 20 and 35 $\mathrm{mmHg},{ }^{5)}$ and patients who had COPD with mPAP greater than $35-40 \mathrm{mmHg}$ at rest were approximately only $1 \%$ $5 \%$ of the population. ${ }^{7,8)}$ In the present report, our patient had GOLD stage III disease with severe PH (out-ofproportion $\mathrm{PH}$ ), which was higher than expected based on the COPD severity. He did not respond to the COPD therapeutic drugs and died of progressive $\mathrm{PH}$ and right heart failure. After the postmortem autopsy, he was finally diagnosed as PVOD complicated with COPD.

Severe $\mathrm{PH}$ is uncommon in patients with COPD; Chaouat, et al. reported that 27 patients $(2.7 \%)$ among 998 patients with COPD developed severe PH (mPAP $\geq$ $40),{ }^{6}$ of whom 16 had another disease capable of causing severe PH, but none was diagnosed with PVOD. The Fifth World Symposium on Pulmonary Hypertension suggested some specific diagnostic criteria to aid clinicians in the differential diagnosis between group 1 and $3 \mathrm{PH}$. The presence of mild airflow limitation (FEV1.0\%, > 60\%), mild or missing lung parenchymal abnormalities on highresolution chest $\mathrm{CT}$, and a primary cardiovascular limitation found during cardiopulmonary exercise testing favor classification into group $1(\mathrm{PAH})$, whereas more severe airflow limitation (FEV1.0\%, <60\%), significant lung parenchymal abnormalities on high-resolution chest $\mathrm{CT}$, and a ventilatory limitation during exercise testing favor group 3 PH. ${ }^{9}$ Accordingly, our patient was diagnosed with group $3 \mathrm{PH}$; however, he was resistant to the treatment of COPD, and the postmortem autopsy finally demonstrated the coexistence of PVOD and COPD, and the lack of histological patterns of severe $\mathrm{PH}$ caused by COPD including arteriole muscularization. ${ }^{10)}$ These observations suggest that the main cause of severe $\mathrm{PH}$ in the present case was PVOD.

PVOD is a rare type of $\mathrm{PH}$ characterized by an obstruction, stenosis, and remodeling of the pulmonary venules. It is subcategorized as group 1' within the current $\mathrm{PH}$ classification. ${ }^{11}$ The etiology is not fully understood, and a variety of risk factors have been described: human immunodeficiency virus infection, genetic factors, autoimmune disorders, and toxic exposures. ${ }^{12)}$ Because of its rarity and similarity with idiopathic PAH, PVOD is difficult to diagnose antemortem and has a poor prognosis. The in- 


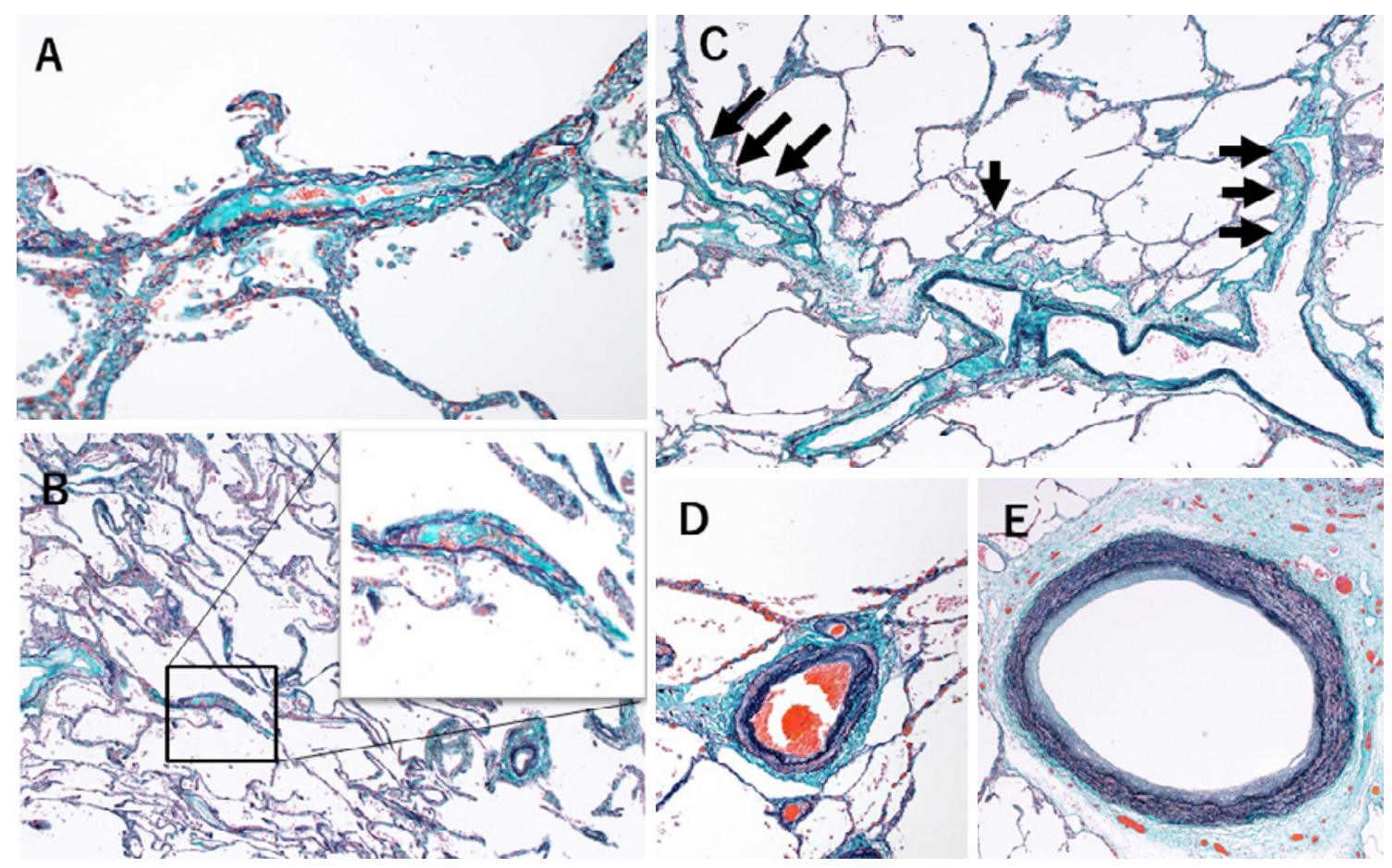

Figure 3. Autopsy specimens of pulmonary vessels on Masson trichrome staining. A, B: Marked intimal fibrous thickening with resulting luminal occlusion in pulmonary intralobular veins. C: Intimal fibrous thickening of pre-septal venules (arrows). D, E: Pulmonary arterial remodeling including increased medial wall thickness and abnormal proliferation of intima; vessel stenosis was not remarkable.

cident rate is $0.2-0.5$ cases per million inhabitants per year, ${ }^{13)}$ and that is estimated to account for $5 \%-10 \%$ of cases diagnosed with group $1 \mathrm{PH} .{ }^{14)}$ One-year mortality is reportedly $72 \%,{ }^{15}$ ) and death typically occurs within 2 years of diagnosis. ${ }^{14)}$ There is no established medical treatment for PVOD, and the only curative therapy is lung transplantation. ${ }^{16)}$ PVOD was designated as an intractable disease in addition to PAH in Japan in 2015. A definite diagnosis of PVOD requires histological analysis of a lung sample, in which extensive and diffuse occlusion of pulmonary veins by fibrous tissue and intimal thickening of venules and small veins in lobular septa is observed. ${ }^{14)}$ Since PH is a contraindication to lung biopsy due to increased risk for uncontrolled hemorrhage, the development of a non-invasive diagnostic tool has been anticipated. The association study of at least two specific high-resolution CT signs among septal thickening, ground glass, and enlarged lymph nodes revealed a sensitivity of $95.5 \%$ and a specificity of $89 \%$ in identifying patients with PVOD. ${ }^{17)}$ In the present case, the three findings could be detected in the high-resolution $\mathrm{CT}$ at the first admission. Moreover, PVOD has distinctive characteristics of progressive hypoxemia on exertion and markedly reduced DLCO. Montani, et al. reported that $80 \%$ of PVOD patients had a DLCO/VA lower than $55 \%$, compared with that of $21 \%$ in PAH patients. ${ }^{14)}$ The patient had severely reduced exercise tolerance during a 6-minute walk test and DLCO, which his COPD severity alone could not explain.

To the best of our knowledge, only one PVOD patient complicated by COPD and severe $\mathrm{PH}$ was reported ${ }^{18)}$; the patient developed diffuse lung emphysema without air- flow limitation. High-resolution CT showed all three signs in favor of PVOD that differentiate PVOD from PAH, and the patient had very low DLCO and severe hypoxemia. These findings strongly suggested PVOD, which was pathohistologically verified after the lung transplantation. By contrast, our patient had severe airflow limitation, which made an accurate diagnosis of PVOD difficult. Further case reports and comprehensive analysis of COPD patients complicated by severe $\mathrm{PH}$ are needed for early diagnosis and treatment of these patients.

In conclusion, we herein reported a COPD case with severe $\mathrm{PH}$ caused by PVOD. Although severe $\mathrm{PH}$ is uncommon in patients with COPD, PVOD is an important consideration in the differential diagnosis of $\mathrm{PH}$, in which the high-resolution CT findings (septal thickening, ground glass, and enlarged lymph nodes) and severely reduced DLCO are most notable.

\section{Disclosure}

Conflicts of interest: The authors have no conflict of interest to disclose.

\section{References}

1. Simonneau G, Montani D, Celermajer DS, et al. Haemodynamic definitions and updated clinical classification of pulmonary hypertension. Eur Respir J 2019; 53: 1801913.

2. Rabe KF, Hurd S, Anzueto A, et al. Global strategy for the diagnosis, management, and prevention of chronic obstructive pul- 
monary disease: gold executive summary. Am J Respir Crit Care Med 2007; 176: 532-55.

3. Elwing J, Panos RJ. Pulmonary hypertension associated with COPD. Int J Chron Obstruct Pulmon Dis 2008; 3: 55-70.

4. Blanco I, Piccari L, Barberà JA. Pulmonary vasculature in COPD: the silent component. Respirology 2016; 21: 984-94.

5. Weitzenblum E, Chaouat A, Canuet M, Kessler R. Pulmonary hypertension in chronic obstructive pulmonary disease and interstitial lung diseases. Semin Respir Crit Care Med 2009; 30: 458-70.

6. Chaouat A, Bugnet AS, Kadaoui N, et al. Severe pulmonary hypertension and chronic obstructive pulmonary disease. Am J Respir Crit Care Med 2005; 172: 189-94.

7. Blanco I, Tura-Ceide O, Peinado VI, Barberà JA. Updated perspectives on pulmonary hypertension in COPD. Int J Chron Obstruct Pulmon Dis 2020; 15: 1315-24.

8. Andersen KH, Iversen M, Kjaergaard J, et al. Prevalence, predictors, and survival in pulmonary hypertension related to endstage chronic obstructive pulmonary disease. J Heart Lung Transplant 2012; 31: 373-80.

9. Kovacs G, Agusti A, Barberà JA, et al. Pulmonary vascular involvement in chronic obstructive pulmonary disease. Is there a pulmonary vascular phenotype? Am J Respir Crit Care Med 2018; 198: 1000-11

10. Bunel V, Guyard A, Dauriat G, et al. Pulmonary arterial histologic lesions in patients with COPD with severe pulmonary hypertension. Chest 2019; 156: 33-44.

11. Simonneau G, Gatzoulis MA, Adatia I, et al. Updated clinical classification of pulmonary hypertension. J Am Coll Cardiol 2013; 62: D34-41.

12. Mandel J, Mark EJ, Hales CA. Pulmonary veno-occlusive disease. Am J Respir Crit Care Med 2000; 162: 1964-73.

13. Szturmowicz M, Kacprzak A, Szołkowska M, Burakowska B, Szczepulska E, Kuś J. Pulmonary veno-occlusive disease: pathogenesis, risk factors, clinical features, and diagnostic algorithm-state of the art. Adv Respir Med 2018; 86: 131-41.

14. Montani D, Achouh L, Dorfmüller P, et al. Pulmonary venoocclusive disease: clinical, functional, radiologic, and hemodynamic characteristics and outcome of 24 cases confirmed by histology. Medicine 2008; 87: 220-33.

15. Holcomb BW, Loyd JE, Ely EW, Johnson J, Robbins IM. Pulmonary veno-occlusive disease: a case series and new observations. Chest 2000; 118: 1671-9.

16. Galie N, Humbert M, Vachiery J, et al. ESC/ERS guidelines for the diagnosis and treatment of pulmonary hypertension. Rev Esp Cardiol 2015; 69: 177.

17. Mineo $\mathrm{G}$, Attinà $\mathrm{D}$, Mughetti $\mathrm{M}$, et al. Pulmonary venoocclusive disease: the role of CT. Radiol Med 2014; 119: 66773.

18. Faure M, Gomez E, Dorfmüller P, et al. A diffuse lung emphysema, severe pulmonary hypertension and lack of airflow limitation. Respir Med Case Rep 2017; 21: 74-7. 